\title{
Changes in hormones, melatonin and cortisol, related to the psychological and sleep states of high school students.
}

\author{
Minjoon Yu${ }^{\text {* }}$, Hyojae $\mathrm{Kim}^{2}$, Youngjae Kim², Jiyong Kim² \\ ${ }^{1}$ Seoseok High School, Gwangju, Korea \\ ${ }^{2}$ Korea International High School in Jeju, Korea \\ *Correspondence to: Minjoon Yu, Seoseok High School, Gwangju, Korea, Tel: +82-360-3800; E-mail: \\ ymj214@naver.com
}

Received date: November 19, 2017; Accepted date: November 28, 2017; Published date: December 05, 2017

Citation: Yu M, Kim H, Kim Y, et al. Changes in hormones, melatonin and cortisol, related to the psychological and sleep states of high school students. J Clin Dentistry Oral Health. 2017;1(2):1-8.

Copyright: (C) $2017 \mathrm{Yu} \mathrm{M}$, et al. This is an open-access article distributed under the terms of the Creative Commons Attribution License, which permits unrestricted use, distribution, and reproduction in any medium, provided the original author and source are credited.

\begin{abstract}
Background: Emotionally and physically unstable high school students in adolescence tend to experience psychological and sleep disturbances in their uniform and stereotyped life. Hence, we investigated the actual condition of the depressive mood and sleep disturbances of high school students. In addition, we made a study on the correlation of cortisol and melatonin hormone concentration to their depression and sleep disturbances.

Methods: We used a questionnaire on depressive moods and a questionnaire on sleep quality respectively to conduct a survey on psychological and sleep states. Melatonin and cortisol concentration in saliva sampled at 7 am and $11 \mathrm{pm}$ were measured by ELISA (Enzyme-Linked Immunoassay), and statistical analysis was performed with SPSS-version 21.

Results: According to the survey of 39 first-year and second-year high school students, there were $38.5 \%$ of the students with a depressive mood and $69.2 \%$ of students with sleep disturbance. Sleepdisturbances students suffered from depressive moods more than normal-sleep student and depressivemood students showed a significant increase in cortisol at 7 am (wake-up time). In addition, students with depressive moods and students with sleep disturbances have showed a significant increase in melatonin at $11 \mathrm{pm}$. There was no grade-specific difference.

Conclusion: In conclusion, Korean high school students frequently suffer from depressive moods and sleep disturbances, and these students also showed changes in the concentration of related hormones, cortisol and melatonin. In particular, students with depressive moods experience a "phase delayed" phenomenon in which their normal circadian rhythms are delayed. This suggests that high school students' psychological and sleep disturbances may also affect hormones in their bodies. Therefore, it is necessary to take measures to reduce psychological and sleep disturbances.
\end{abstract}

Keywords: Depressive mood, Sleep disturbance, Saliva, Cortisol, Melatonin, High school student.

\section{Introduction}

The human body has a circadian rhythm according to the repetitive daily biological cycle. This is essential for survival, but the quality of life can depends on its change. It can also be called a biological regulation factor, and it is now known that the biological clock is used for physiological functions (e.g. body temperature, hormone synthesis and sleep-arousal cycle) and psychological functions (e.g. cognitive functions and memory power). A biological rhythm, which is a diurnal $24 \mathrm{~h}$ cycle, shows a difference in behavior and vital functions, depending on the day-night change [1].
A biological rhythm, also called bio-rhythm, is a biological clock which is endogenous in the body. This biological clock works spontaneously and is genetically inherent. Since the variability of the circadian rhythm is affected by the light-dark cycle (e.g. light and darkness) and the external environment (e.g. temperature and weather), its cycle can be altered or reset. Ultimately, it can be said that this biological clock is responsible for controlling the homeostasis in the human body. Earlier studies on human circadian rhythms have suggested that they are affected by changes in light related to the Earth's rotation. It has been known that the suprachiasmatic nucleus in the brain hypothalamus is responsible for the cycle stimulated by light [2]. 
Citation: Yu M, Kim H, Kim Y, et al. Changes in hormones, melatonin and cortisol, related to the psychological and sleep states of high school students. J Clin Dentistry Oral Health 2017;1(2):1-8.

However, it has been suggested that there are other types of regulation factors because circadian rhythms are maintained in the light-free space or by the blind [3]. In order to support such research, there have been studies on how changes in circadian rhythms caused by changes in life patterns (e.g. age, lifestyle, working style, etc.) affect the physical and psychological responses.

The results of these studies are summarized as follows: the lower social rhythm stability of the elderly directly affects their depression, and the nurses or male workers who works at night and sleeps during the day suffer from sleep disturbances and increased blood pressure at night [4-6]. In this context, Korean adolescents who go to school early in the morning and come home from school late in the evening due to their academic work may suffer from various sleep and psychological problems because of changes in their circadian rhythms.

Adolescence is a transition period to adulthood, accompanied by emotional, cognitive, and physical instability. These changes can have both good and bad effects on ego-formation [7]. Adolescence are most appropriately expressed as 'a period of storm and stress' because this period is followed by a series of confusion and tension [8]. In this transitional period, adolescents who lack social knowledge and experience are more vulnerable to stress than adults. If they do not resolve conflicts and confusion, they will experience mental illness such as depression, bipolar disorder and sleep disturbance [9]. In addition, unlike other countries, Korean adolescents may be stressed not only by the developmental changes experienced during this period but also by the burden of excessive academic work due to the competition for university entrance. However, high school students, who spend most of their lives at school, may not be able to grasp their own problems under uniform guidance.

Adolescent depression is important because of their severe masked depression [10]. Masked depression refers to a form of depression hidden under the wearing mask, and it does not reveal the typical symptoms of depression even though depression is developing. The depressive symptoms of adolescents are different from the direct depressive symptoms of adults. That is, adolescents suddenly get angry and irritated or continuously show their sad feelings. They also show truancy, lower school performance, increased sleep, and difficulties in relationships with peers. Therefore, not only adolescents but also people around them are unaware of depression in many cases [11]. If these symptoms persist, appetite loss, anxiety disorders, sleep disturbances and psychosomatic disorders may also occur. Such overlooked adolescent depression can lead to major depressive illness in adulthood and cause serious problems [12]. According to the data released by the Ministry of Health and Welfare in 2009, the prevalence of depression in adolescents increased from $29.9 \%$ in 2005 to $41.3 \%$ in 2007 [13]. The rapid increase in the prevalence of adolescent depression caused by the following reasons: sudden physical changes with adolescence growth, as well as academic stress, uncertainty about their future, and school life where all the focus is on the college entrance examination. According to the survey on academic stress, it increased from $57.1 \%$ in 1998 to $67 \%$ in 2006 [14].

Sleep an essential survival method for preserving the homeostasis and energy of the human body since it allows people to relieve daytime fatigue and rest at night [15]. These sleep-arousal factors are associated with circadian rhythms.

That is, they are awake during the day and become drowsy in the evening so that the rhythm of life is maintained. The optimal health condition can be maintained by harmonizing changes in the body's physiological cycle with the external environment. Melatonin is the hormone most important to sleep. It can be an effective treatment for 'delayed sleep phase syndrome' and 'advanced sleep phase syndrome' caused by a dysregulation of a person's circadian rhythm [16,17]. Here, the delayed sleep phase syndrome refers to 'late to bed, late to rise', and the advanced sleep phase syndrome refers to 'early to bed, early to rise' In particular, adolescent sleep plays a key role in brain growth and development, and it also is very important to maintain cognitive development, emotional health and immunity [18].

These adolescent sleep disturbances can prohibit adolescents from living their normal daily lives because of unpleasant moods, concentration problems and excessive daytime drowsiness. Adolescents with sleep disturbances may also experience memory disorders and lack of social skills in interpersonal relationships [19].

Moreover, adolescence has the highest amount of melatonin secretion at night in their lifetimes. In addition to the social and school problems, sleep disturbances due to hormonal imbalance can become more severe when their academic work makes them cyclically sleep late and get up early in the morning [20]. In particular, sleep begins in 1-2 $\mathrm{h}$ after melatonin secretion and ends in 1-2 $\mathrm{h}$ before stopping the secretion. Thus, the theoretical rationale for identifying melatonin's circadian rhythm to help the treatment of sleep disturbances is formed.

In addition, stress and external stimuli cause hypothalamuspituitary-adrenal axis (HPA axis) abnormalities which affect the secretion of cortisol. This has to do with the development of depressive disorders. The normal in-body response is to activate the autonomic nervous system (ANS) and the endocrinologic functions through the hypothalamus by delivering stress stimuli to the reticular activating system and the thalamus. As a result, cortisol is secreted [21].

However, the normal diurnal variation in which cortisol concentration decreases in the evening more than in the morning. However, patients with depressive disorders shows reversed diurnal variation in which the concentration increases in the evening [22]. In addition, the greater the degree of depression gets, the greater the concentration of cortisol gets in the evening. Therefore, cortisol may be used as a good indicator of the therapeutic effect during treatment of depressive disorders [23]. In some cases, cortisol concentration may increase in the morning because of the uncontrollable or unpredictable future stress and anxiety after people wake up [24]. 
Several studies have shown a close relationship between depression and sleep disturbances. Although there have been various studies on the anteroposterior relationship between the two aspects, the results are diverse and may be disagreed [25]. However, there is no question that they can be deteriorating factors for each other. In addition, students with depressive symptoms caused by sleep disturbances suffer from depression longer than students without depressive symptoms [26]. Sleep disturbances and depressive symptoms are influenced by several factors: region, family, school, and friendship. Of course, there is a difference in prevalence between men and women. It is generally said that girls have a higher prevalence [27].

Although the recommended adolescent sleep time is $8 \mathrm{~h}$, the average weekly sleeping time of Korean high school students is $5.4 \mathrm{~h}$, which is less than $7-8 \mathrm{~h}$ in the US, $7.5 \mathrm{~h}$ in China and 6-7 h in Japan. Thus, many students suffer from chronic sleep deprivation [28]. If such sleep deprivation persists, it causes physical imbalance, adolescent developmental disorders, and mood and emotional problems which lead to depressed mode. If such problem is not resolved and continues this problem continues in a vicious cycle, a treatment such as medication is required [29].

As mentioned above, it is true that the school life and social life of Korean high school students are hindered by various variable factors. In particular, if they mentally or physically grow in the wrong direction during their immature adolescence, there may be irreversible consequences when they become adults. Therefore, it is important to identify problems related to current adolescents and to change the variable factors if possible. First, it is necessary to study the psychology, body and sleep quality of high school students and to identify the relationship between psychology used as a biological indicator and hormones related to sleep. In this study, we aim at investigating the psychological and sleep states of Korean high school students and at measuring melatonin (a sleeping hormone) and cortisol (a stress hormone) to determine whether changes in hormone concentrations are related to sleep and psychological states. Furthermore, to understand their mental health problems, we intend to present the base data useful for developing measures to improve their mental health and physical health in the future.

\section{Methods}

\section{Research subjects}

In this study, we conducted a cross-sectional study on first and second-year high school students. Data were collected from June, 2017 to July, 2017. All questionnaires were collected in a one-to-one face-to-face way. We used the following research three tools: a questionnaire about basic information, Pittsburgh Sleep Quality Index (PSQI), and Zung Self-Rating Depression Scale. Melatonin and cortisol in saliva were measured by an external testing agency. Among the total 60 students, an analysis of 39 students were finally made, except for 21 students who didn't complete questionnaires accurately or whose saliva samples are not enough to get their test results.
The research subjects include 25 first-year high school students and 14 second-year high school students.

\section{Research tools}

Questionnaire form: The questionnaire about basic information: We have surveyed the grades, ages, heights, and weights of the students to understand their basic information.

Depression mood screening tool: There are various questionnaires about depression mood screening. In this study, we used the most widely used Zung Self-rating Depression Scale (ZDS) developed by Zung in 1965. This questionnaire consists of 20 questions, Each question is scored on a scale of 1 through 4 , and the scores range up to 80 points $(1=$ some of the time, $2=$ some of the time, $3=$ good part of the time, $4=$ most of the time). The total score indicates the level of depression. In a psychological aspect, the worst score is 80 and the best score is 20. The scores is divided into the following four ranges: 20-44 (normal range), 45-59 (mildly depressed), 60-69 (moderately depressed), 70 and above (severe depressed). In this study, we compared the normal group (20-44) and the depressed group (45 or above).

Sleep pattern measurement tool: Even with the same sleeping time, how much of the body's function is restored during sleep depends on the quality of sleep which allows the body to rest. In this study, we used the Pittsburgh Sleep Quality Index (PSQI) developed by Buysse in 1989 to assess sleep quality. The PSQI is the most widely used questionnaire for measuring sleep quality, and its reliability and validity have been proven by many studies. Therefore, we adopted the PSQI for this study. The questionnaire which consists of 10 question items measures sleep quality and disturbances over a 1-month time interval. The measure consists of 24 individual question items including the sub-questions. Sleep quality can be expressed by 7 components: subjective sleep quality, sleep latency, sleep duration, habitual sleep efficiency, sleep disturbances, use of sleep medication, and daytime dysfunction. Each component is weighted on a $0-3$ interval scale, and an overall score ranges up to 21 , where lower scores denote a healthier sleep quality. In this study, the students were divided into the following two groups on the basis of PSQI scores: the normal-sleep group (5 or below) and the sleepdisturbance group (6 or above).

\section{How to measure diurnal hormones?}

Precautions for sampling Saliva: Limit Alcohol, Caffeine and Nicotine intake in $12 \mathrm{~h}$ before sampling. Do not eat or drink anything in $1 \mathrm{~h}$ before sampling. Do not brush your teeth in $45 \mathrm{~min}$ before sampling. Rinse your mouth with cold water $10 \mathrm{~min}$ before sampling.

1. Do not take samples in case of oral disease, inflammation or lesions because the samples may be contaminated with blood.

2. Visually check whether the red blood is mixed with the saliva samples.

3. Use saliva collection tubes for measuring salivary cortisol, salivary alpha-amylase and salivary testosterone, and 
Citation: Yu M, Kim H, Kim Y, et al. Changes in hormones, melatonin and cortisol, related to the psychological and sleep states of high school students. J Clin Dentistry Oral Health 2017;1(2):1-8.

allow the saliva samples to penetrate the cotton in the container.

4. For steroid testing, use flowing saliva samples in sterile tubes. (Salivary DHEA, Salivary IgA, etc.)

5. Do not touch the cotton with your bare hands.

Saliva sampling time and sample management: Saliva was sampled twice a day, at 11 p.m. and 7 a.m., and it was ensured that the minimum amount of each sample was $0.3 \mathrm{ml}$. Each saliva sample was immediately refrigerated, and all the collected samples are analyzed by an external testing agency.

\section{How to test melatonin and cortisol in saliva?}

The collected saliva samples are examined and analyzed by an external testing agency as follows. First, to test salivary melatonin, a buffer solution is pretreated several times by performing an Enzyme Immunoassay (EIA) through the capture antibody reaction, and then the melatonin-biotinantibody complexes were used to detect the antibody bound to melatonin using an absorbance at $450 \mathrm{~nm}$ (The BÜHLMANN Direct Saliva Melatonin ELISA, Switzerland). To test salivary cortisol, an absorbance microplate reader (V Max 220 VAC, Molecular Devices, CA, USA) and an Enzyme Immunoassay kit (HS salivary cortisol EIA kit, Salimetrics, PA, USA) were used for performing an Enzyme Immunoassay (EIA). Like salivary melatonin, an absorbance at $450 \mathrm{~nm}$ is used for detecting the antibody.

\section{How to do statistical analysis?}

We analysed the descriptive statistics of students' basic information and each item respectively, and we provided the calculated average, standard deviation, standard error, maximum value, minimum value, etc. We performed a ChiSquare test to test the relationship of sleep disturbances with the normal-mood group and depressive-mood groups. Differences in cortisol and melatonin concentrations between the two groups were analyzed by mean comparison (MannWhitney $U$ test). In addition, we performed a simple correlation analysis (Peason's correlation) to check the correlation of depression and sleep disturbance scores with each hormone concentration. The statistical significance of a test result was defined as the p-value less than or equal to 0.05 .

\section{Results}

\section{Survey of the high school students on their general characteristics and life patterns}

General characteristics: All the high school students surveyed had the following characteristics: the average age was $17.15 \pm 0.36$, the body weight was $61.08 \pm 10.41 \mathrm{~kg}$, and the height was $172.2 \pm 5.54 \mathrm{~cm}$. There were 25 first-year high school students with the following characteristics: the average age was $17 \pm 0.00$, the body weight was $61.67 \pm 13.02 \mathrm{~kg}$, and the height was $173.2 \pm 6.05 \mathrm{~cm}$. There were 14 second-year high school students with the following characteristics: the average age was $17.43 \pm 0.51$, the body weight was $62.07 \pm$ $5.81 \mathrm{~kg}$, and the height was $170.1 \pm 3.95 \mathrm{~cm}$ (Table 1).

Table 1: A comparison of general characteristics, sleep and psychological states among all target students and grade-specific students [ (¹): Zung self rating depression scale; ( 2): Pittsburgh sleep quality index; (3): Melatonin; ( 4): Cortisol].

\begin{tabular}{|c|c|c|c|c|c|c|c|c|c|c|}
\hline & & \multirow{3}{*}{ Age } & \multirow{3}{*}{ Weight (kg) } & \multirow{3}{*}{$\begin{array}{l}\text { Height } \\
\text { (cm) }\end{array}$} & \multirow{3}{*}{ ZDS *1 } & \multirow{3}{*}{ PSQI 2} & \multirow{2}{*}{$\frac{\mathrm{mel}^{*} 3}{11 \mathrm{PM}}$} & \multirow{2}{*}{$\begin{array}{c}\text { mel } \\
7 \mathrm{AM}\end{array}$} & \multirow{2}{*}{$\begin{array}{c}\text { Corti *4 } \\
11 \mathrm{PM}\end{array}$} & \multirow{2}{*}{$\begin{array}{l}\text { corti } \\
7 A M\end{array}$} \\
\hline & & & & & & & & & & \\
\hline & & & & & & & $(\mathrm{pg} / \mathrm{ml})$ & (pg/ml) & $(\mu \mathrm{g} / \mathrm{dl})$ & $(\mu \mathrm{g} / \mathrm{dl})$ \\
\hline & Total & 17.15 & 61.08 & 172.2 & 38.64 & 8.44 & 7.611 & 2.266 & 0.035 & 0.292 \\
\hline \multirow[t]{3}{*}{ Average } & 1st grade & 17 & 61.67 & 173.2 & 35.92 & 7.56 & 6.905 & 2.256 & 0.038 & 0.291 \\
\hline & 2 nd grade & 17.43 & 62.07 & 170.1 & 43.5 & 10 & 8.871 & 2.284 & 0.029 & 0.295 \\
\hline & Total & 0.366 & 10.41 & 5.54 & 7.81 & 3.09 & 6.795 & 1.559 & 0.018 & 0.147 \\
\hline \multirow{3}{*}{$\begin{array}{l}\text { SD (standard } \\
\text { Deviation) }\end{array}$} & 1st grade & 0 & 13.02 & 6.05 & 7.42 & 2.66 & 6.68 & 1.59 & 0.018 & 0.149 \\
\hline & 2 nd grade & 0.51 & 5.81 & 3.95 & 6.06 & 3.28 & 6.164 & 0.987 & 0.015 & 0.103 \\
\hline & Total & 0.059 & 1.666 & 0.89 & 1.25 & 0.49 & 1.359 & 0.311 & 0.003 & 0.029 \\
\hline \multirow[t]{2}{*}{ SE (standard Error) } & 1st grade & 0 & 2.66 & 1.24 & 1.48 & 0.53 & 1.4 & 0.324 & 0.003 & 0.03 \\
\hline & 2 nd grade & 0.14 & 1.552 & 1.06 & 1.619 & 0.88 & 1.647 & 0.263 & 0.004 & 0.027 \\
\hline$P$ value & & & & & 0.073 & 0.099 & 0.377 & 0.952 & 0.147 & 0.946 \\
\hline
\end{tabular}

The results of normal-mood and depressive-mood states measured by ZDS: Among the total 39 high school students, 24 students $(61.5 \%)$ were normal and 15 students $(38.5 \%)$ were depressed. The average ZDS score was $38.64 \pm 7.81$ in the normal-mood group and $47.06 \pm 2.52$ in the depressivemood group, respectively. The average ZDS of first-year high school students and that of second-year high school students were $35.92 \pm 7.42$ and $43.50 \pm 6.06$, respectively, but there was 
no statistical significance $(\mathrm{P}=0.073)$. In the depressive-mood group, there were $7(28 \%)$ out of 25 first-year high school students with an average ZDS score of 45.86 and $8(57.1 \%)$ of 14 second-year high school students with an average ZDS score of 48.13 . The ZDS score of second-year high school students was slightly higher than that of the first-year high school students, but there was no statistical significance $(\mathrm{P}>0.05)$ (Table 1 and 2).

Table 2: Comparison of sleep quality, melatonin and cortisol levels between the normal mood group and the depressive mood group; [( 1$)$ : Zung self rating depression scale; ( 2$)$ : Pittsburgh sleep quality index; ( 3): Melatonin; ( 4 ): Cortisol].

\begin{tabular}{|c|c|c|c|c|c|c|}
\hline & & \multirow{3}{*}{ ZDS ${ }^{*} 1$} & \multirow{3}{*}{ PSQI ${ }^{*} 2$} & mel *3 & Corti ${ }^{*} 4$ & corti \\
\hline & & & & 11PM & 11PM & $7 A M$ \\
\hline & & & & (pg/ml) & $(\mu \mathrm{g} / \mathrm{dl})$ & $(\mu \mathrm{g} / \mathrm{dl})$ \\
\hline \multirow{2}{*}{ Average } & Normal & 33.37 & 7.25 & 3.739 & 0.039 & 0.239 \\
\hline & $\begin{array}{l}\text { Depressive } \\
\text { group }\end{array}$ & 47.06 & 10.33 & 13.806 & 0.028 & 0.377 \\
\hline \multirow{2}{*}{$\begin{array}{l}\text { SD } \\
\text { (standard } \\
\text { Deviation) }\end{array}$} & Normal & 9.186 & 3.193 & 1.916 & 0.017 & 0.083 \\
\hline & $\begin{array}{l}\text { Depressive } \\
\text { group }\end{array}$ & 2.52 & 1.72 & 6.639 & 0.015 & 0.152 \\
\hline \multirow{2}{*}{$\begin{array}{l}\text { SE } \\
\text { (standard } \\
\text { Error) }\end{array}$} & Normal & 0.947 & 0.651 & 0.391 & 0.003 & 0.017 \\
\hline & $\begin{array}{l}\text { Depressive } \\
\text { group }\end{array}$ & 0.65 & 0.44 & 1.714 & 0.004 & 0.039 \\
\hline$P$ value & & 0.001 & 0.001 & 0 & 0.055 & 0.001 \\
\hline
\end{tabular}

The results of normal sleep and sleep disturbance states measured by PSQI: Among the total 39 high school students, 12 students $(30.8 \%)$ had normal sleep and 27 students $(69.2 \%)$ have sleep disturbances. The average PSQI of the normal-sleep group and that of the sleep-disturbance group were $4.75 \pm 0.62$ and $10.07 \pm 2.18$, respectively. Therefore, there was a statistically significant difference between the two group $(p=0.001)$. Taking a close look at first-year high school students and second-year high school students concerning sleep-disturbances revealed the following results. There were $15(62.5 \%)$ first-year high school students with an average PSQI score of $7.56 \pm 2.66$ and $12(85.7 \%)$ second-year high school students with an average PSQI score of $10.0 \pm 3.28$. However, there was no significant difference $(\mathrm{P}=0.099)$. That is, given the high school students, there was no grade-specific difference in sleep quality but they generally suffered from severe sleep disturbances. In addition, the ZDS score of the normal sleep group and that of the sleep-disturbance group is $33.08 \pm 4.25$ and $41.11 \pm 7.80$, respectively. That is, the depression of the sleep-disturbance group was more severe than that of the normal sleep group. There was a statistically significant difference, and the correlation between sleep disturbances and depression was found $(\mathrm{P}=0.001)$ (Table 3$)$.

\section{The correlation between melatonin changes and cortisol changes in high school students}

Correlation between grade-specific changes: The methods for measuring the two hormones and the reference values used for analyzing the test results varied depending on each institute. In this study, the normal levels of melatonin depending on cardinal rhythm were defined as follows: 7-9 am $<10.50 \quad(\mathrm{pg} / \mathrm{ml}), 3-5 \mathrm{pm}<0.88 \quad(\mathrm{pg} / \mathrm{ml})$, and 2-3 am $=2.53 \sim 30.67(\mathrm{pg} / \mathrm{ml})$, the normal levels of cortisol were defined as follows: $0.021 \sim 0.883(\mu \mathrm{g} / \mathrm{dl})$ in the morning and $0 \sim 0.259(\mu \mathrm{g} / \mathrm{dl})$ in the evening. Given the entire high school students surveyed, the average levels of melatonin were 7.611 $\pm 6.795(\mathrm{pg} / \mathrm{ml})$ at $11 \mathrm{pm}$ and $2.266 \pm 1.559(\mathrm{pg} / \mathrm{ml})$ at $7 \mathrm{am}$.

Table 3: Comparison of general characteristics, sleep and psychological states, melatonin and cortisol levels between the normal sleep group and the sleep disturbance group; [( 1): Pittsburgh sleep quality index; ("2): Zung self rating epression scale; (*3): Melatonin; ( 4); Cortisol]

\begin{tabular}{|c|c|c|c|c|c|c|c|c|c|}
\hline & & \multirow{3}{*}{ PSQI *1 } & \multirow{3}{*}{ Age } & \multirow{3}{*}{ Weight (kg) } & \multirow{3}{*}{ Height $(\mathrm{cm})$} & \multirow{3}{*}{$\mathrm{ZDS}^{*} 2$} & \multirow{2}{*}{$\begin{array}{c}\text { Mel }^{\star} 3 \\
\text { 11PM }\end{array}$} & \multirow{2}{*}{$\begin{array}{r}\text { Corti*4 } \\
\text { 11PM }\end{array}$} & \multirow{2}{*}{$\begin{array}{l}\text { corti } \\
7 A M\end{array}$} \\
\hline & & & & & & & & & \\
\hline & & & & & & & (pg/ml) & $(\mu \mathrm{g} / \mathrm{dl})$ & $(\mu \mathrm{g} / \mathrm{dl})$ \\
\hline \multirow{2}{*}{ Average } & Normal & 4.75 & 17 & 62 & 173 & 33.08 & 3.171 & 0.029 & 0.256 \\
\hline & Sleep disturbance & 10.07 & 17.22 & 61.76 & 171.6 & 41.11 & 9.585 & 0.038 & 0.31 \\
\hline \multirow{2}{*}{$\begin{array}{l}\text { SD (standard } \\
\text { Deviation) }\end{array}$} & Normal & 0.62 & 0 & 15.65 & 8.26 & 4.25 & 1.12 & 0.013 & 0.093 \\
\hline & Sleep disturbance & 2.18 & 0.42 & 8.24 & 4.1 & 7.8 & 7.02 & 0.019 & 0.144 \\
\hline \multirow{2}{*}{ SE (standard Error) } & Normal & 0.18 & 0 & 4.95 & 2.49 & 1.23 & 0.323 & 0.004 & 0.027 \\
\hline & Sleep disturbance & 0.42 & 0.08 & 1.65 & 0.79 & 1.5 & 1.351 & 0.004 & 0.028 \\
\hline$P$ value & & 0.001 & & & & 0.001 & 0 & 0.148 & 0.244 \\
\hline
\end{tabular}

Given the first-year and second-year high school students, the average levels of melatonin at $11 \mathrm{pm}$ were $6.905 \pm 6.680$ and $8.871 \pm 6.164$ respectively $(\mathrm{P}=0.377)$, and the average levels of melatonin at 7 am were $2.256 \pm 1.590$ and $2.284 \pm 0.987$ respectively $(\mathrm{P}=0.952)$. There was no significant difference between the two groups at both time points. The average levels of cortisol at $11 \mathrm{pm}$ were $0.038 \pm 0.018$ and $0.029 \pm 0.015$ respectively $(\mathrm{p}=0.147)$, and the average levels of cortisol at 7 am were $0.291 \pm 0.149,0.295 \pm 0.103$ respectively $(\mathrm{p}=0.946)$. There was also no significant difference. Melatonin and 
Citation: Yu M, Kim H, Kim Y, et al. Changes in hormones, melatonin and cortisol, related to the psychological and sleep states of high school students. J Clin Dentistry Oral Health 2017;1(2):1-8.

cortisol showed high hormonal changes at $11 \mathrm{pm}$ and at 7 am respectively, but there was no grade-specific difference (Table $1-3)$.

Changes in melatonin and cortisol depending on psychological states: The melatonin levels of the normalmood and depressive-mood groups at $11 \mathrm{pm}$ were $3.739 \pm$ 1.916 and $13.806 \pm 6.639$, respectively $(\mathrm{P}=0.000)$. There was a significant difference between two groups. In comparison to the circadian melatonin rhythm, this result was consistent with a "phase delayed" phenomenon observable in depressed patients [26].

The cortisol levels of the normal-mood and depressive-mood groups at $11 \mathrm{pm}$ were $0.039 \pm 0.017$ and $0.028 \pm 0.015$ respectively $(\mathrm{P}=0.055)$; thus, there was no significant difference. On the other hand, the cortisol levels of the normalmood and depressive-mood groups at 7 am were $0.239 \pm 0.083$ and $0.377 \pm 0.152$ respectively $(\mathrm{p}=0.001)$; thus, there was a significant difference. These results indicated that the depressive-mood group had a higher cortisol level than the normal-mood group. In conclusion, there may be changes in circadian hormones depending on psychological moods. In this study, melatonin and cortisol showed a higher hormonal change at $11 \mathrm{pm}$ and at 7 am respectively in depressive-mood students (Table 2), (Figure 1). Given all the high school students surveyed, there was a significant correlation between melatonin (11 pm) and cortisol (7 am), depending on their psychotic states (ZDS score) (Figure 1). These results suggest that depression may affect hormonal changes in the body.
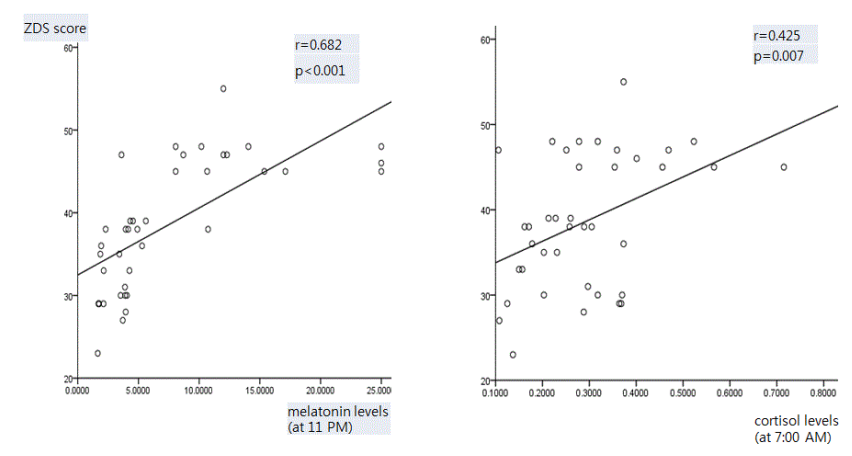

Figure 1. Relationship of melatonin (11 pm) and cortisol (7 am) concentration to psychological states (ZDS score)-By Peason's correlation.

Changes in melatonin and cortisol depending on sleep quality: Given all the students surveyed, there was a significant correlation between melatonin concentration (11 pm) and sleep quality (PSQI score) (Figure 2).

The melatonin levels of the normal-sleep and sleep-disturbance groups at $11 \mathrm{pm}$ were $3.171 \pm 1.120$ and $9.585 \pm 7.020$, respectively $(\mathrm{P}<0.001)$. There was a significant difference between the two groups. These results suggest that there is a correlation between sleep quality and melatonin concentration in the body.

The average cortisol levels of the normal-sleep and sleepdisturbance groups at $11 \mathrm{pm}$ were $0.029 \pm 0.013$ and $0.038 \pm$ 0.019 respectively $(\mathrm{P}=0.148)$. The average cortisol levels of the normal-sleep and sleep-disturbance groups at 7 am were 0.256 \pm 0.093 and $0.310 \pm 0.144(\mathrm{p}=0.244)$, respectively. There was no statistically significant difference. Regarding hormonal changes depending on sleep quality, melatonin changes turned out to be more noticeable than cortisol changes (Table 3) (Figure 2).

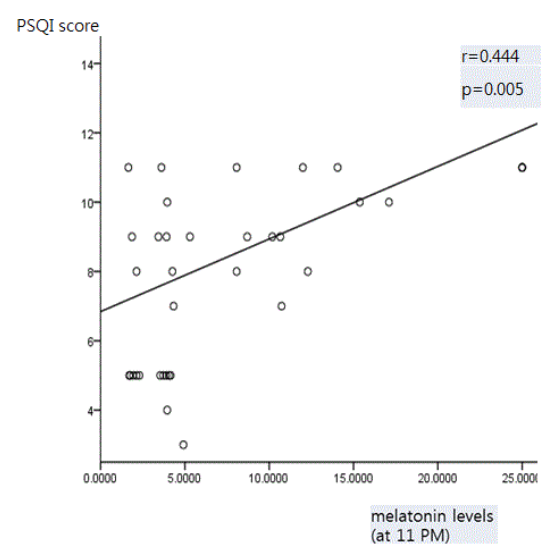

Figure 2. Relationship of melatonin concentration (11 pm) to the sleep state (PSQI score)-By Peason's correlation.

\section{Discussion}

Stress in adolescents is more complicated than adults and difficult to predict the outcome because they experience conflict and confusion due to lack of experience in various real situations [30]. In addition, pressure caused by the competition for entrance examination and academic stress are the most important part of Korean high school students' lives [31]. This study is designed to investigate sleep quality because they suffer from depression caused by such stresses and sleep deprivation caused by the burden of academic work. In addition, it is aimed at finding their in-body psychology and sleep-related hormonal changes in physically immature adolescents. Based on these results, it is expected that this study will be used as base data to find efficient management methods for improving the health of adolescents in the future.

As a result of surveying high school students on depression, $38.5 \%$ of the students showed depressive moods. There was no grade-specific statistical significance. However, $57.1 \%$ of the second-year high school students and $28 \%$ of the first-year high school students showed depressive moods. That is, the number of the depressed second-year high school students was higher than that of the depressed first-year high school students. This result is consistent with the survey result reported by the Seoul School Health Promotion Center showing that the second-year high school boys and the first year high school girls are at the highest level of psychological depression during the whole school year [32].

In the sleep survey, $69.2 \%$ of the total students complained of sleep disturbances. There was a high prevalence rate of sleep disturbances in the first-and second-year high school students, showing $62.5 \%$ and $85.7 \%$, respectively. However, there was no significant grade-specific change in sleep disturbance. This suggests that the problem of sleep disturbance can be a problem for all high school students, regardless of grades. In 
addition, there is a report that sleep disturbances are not a problem of some schools and that the same result is observed in various types of high school in Korea. According to several research reports on sleep disturbances in high school students, $62.1 \%$ of gifted high school students, $83.6 \%$ of science high school students, $80.9 \%$ of general students and $84.9 \%$ of international high school students suffer from sleep disturbances. These results show no significant difference from this study, and it can be known that sleep disturbance is a common problem among all high school students in Korea [33].

We investigated melatonin and cortisol in saliva to observe the effects of depressive mood and sleep disturbance on circadian rhythm. Melatonin is produced in the pineal gland of the brain and regulates sleep $[34,35]$. Its secretion is regulated through the circadian rhythm. It falls to the basal level around 9-10 am in the morning and increases around $10-11 \mathrm{pm}$ in the evening, peaking at $2 \mathrm{am}$ [36]. It is sensitive to light so that it senses sunlight during the day. When the signal is transmitted to the pineal gland, its production is suppressed. Melatonin acts as a zeitgeber that stimulates its production when light is not delivered in the evening. A zeitgeber is any environmental factor that affects an organism's biological rhythms to the cycle in order to make environmental factors undergo periodic changes. Its representative example is melatonin's secretion regulation [36]. In addition, several studies have reported that increasing the production of melatonin by its administration to artificially control the sleep cycle is effective for jet lag and sleep disorder [37-39]. In addition, patients with depression experience a "phase delayed" phenomenon that is overserved later than the normal circadian rhythm. For this reason, they are unable to sleep in the evening, and their sleeping time becomes late, making it difficult for them to wake up in the morning hour, resulting in more drowsiness during the day [40]. This study also shows that students' depressive moods peak at $11 \mathrm{pm}$; therefore, the result of this study is consistent with several reports indicating that the circadian cycle of melatonin is delayed in people suffering from depression. Using this principle, melatonin administered at the appropriate time is suggested as a solution to problems related to sleep and circadian rhythm because it can speed up or slow down the inbody biological clock.

To understand the relationship between sleep and hormones (cortisol and melatonin), we first need to look at the structure of sleep. Non-Rapid Eye Movement (NREM) is divided into four stages. Stage 3 is the deepest sleep referred to as slowwave sleep (SWS) which takes an average of 10-20 min. In this stage, the EEG (electroencephalogram) shows more than $50 \%$ delta waves, a high amplitude wave, and the body temperature, respiration and heart rate are also reduced. Stage 4 is the deepest process of sleep, accounting for $12-15 \%$ of the total sleep. Cortisol is associated with sleep. Thus, slow wave sleep is abundant in early sleep, where cortisol secretion is abruptly suppressed. REM sleep is further activated at the wake-up time in the morning due to the increased cortisol concentration [41]. For this reason, when the circadian rhythm is checked, melatonin and cortisol are reversely secreted. At the peak of melatonin at dawn, the cortisol secretion is decreased, and when the melatonin secretion is decreased in the morning, the secretion of cortisol is increased [42]. In the normal circadian rhythm, the cortisol level decrease rapidly during the daytime and peaks immediately before the wake-up time because of its rapid increase at dawn. In this study, both normal-mood and depressive-mood groups show higher cortisol levels at $7 \mathrm{am}$, In particular, the depressive-mood group show higher cortisol levels at 7 am and show a statistically significant increase along with the normal-mood group. These results are consistent with many reports that the cortisol level may increase further in patients with depressive disorders and that cortisol concentrations in the morning may be further increased by worrying about future events after people wake up $[43,44]$. Regarding hormonal changes depending on sleep quality, the sleep-disturbance group shows a significantly different increase in the melatonin level at $11 \mathrm{pm}$, compared to the normal-sleep group. However, cortisol concentration shows no significant relationship with sleep quality. This indicates that measuring changes in melatonin (a sleeping hormone) is more useful than changes in cortisol (a stress hormone) to examine the therapeutic effect when students with depressive moods and sleep disturbances are treated by considering their seriousness.

\section{Conclusion}

According to the results of the study suggest that a depressive mood and a sleep disturbance are relatively common in adolescents. There was no grade-specific difference. It means that all high school students suffer from such problems. In addition, there is a correlation between sleep and depression. Since these sleep and psychological states may affect hormonal changes in the body, they can affect physical health. Therefore, it is necessary to take measures to cope with the sleep disturbances and depressive moods of adolescents.

\section{Reference}

1. Horne Ja, Östberg O. A self-assesment questionare to determine moriningness-eveningness in human circadian rhythm. Chronobiol Int. 1976;4(2):97-110.

2. Bloom FE, Lazerson A. Brain, mind, and behavior. New York: Freeman. 1998.

3. Minors DS, Waterhouse JM. Circadian rhythms in general. Occup Med. 1990;5(2):165-82.

4. Panda S, Sato TK, Castrucci AM, et al. Melaonopsin requirement for normal light-induced circardian phase shifting. Issues of Journal Science. 2002.

5. Kaliyaperumal D, Elango Y, Alagesan M, et al. Effects of sleep deprivation on the cognitive performance of nurses working in shift. J Clin Diagn Res. 2017;11(8):CC01-3.

6. Ohida T, Takemura S, Nozaki N, et al. The influence of lifestyle and night-shift work on sleep problems among female hospital nurses in Japan. Nippon Koshu Eisei Zasshi. 2001;48(8):595-603.

7. Wallerstein RS. Erikson's concept of ego identity reconsidered. J Am Psychoanal Assoc. 1998;46:229-47. 
Citation: Yu M, Kim H, Kim Y, et al. Changes in hormones, melatonin and cortisol, related to the psychological and sleep states of high school students. J Clin Dentistry Oral Health 2017;1(2):1-8.

8. Hall GS. Adolescence; its psychology and its relationships to physiology, anthropology, sociology, sex, crime, religion and education. New York: Appleton. 1904.

9. Paus T. Mapping brain development and aggression. Can Child Adolesc Psychiatr Rev. 2005;14:10-5.

10. March JS, Silva S, Petrycki S, et al. The Treatment for Adolescents With Depression Study (TADS): Long-term effectiveness and safety outcomes. Arch Gen Psychiatry. 2008;65(1):101.

11. Cantwell DP, Baker L. Manifestations of depression affect in adolescence. J Youth Adolesc. 1991;20:121-33.

12. Tylee A, Gandhi P. The importance of somatic symptoms in depression in primary care. Prim Care Companion $\mathrm{J}$ Clin Psychiatry. 2005; 7:167-76.

13. Ministry of Health and Welfare. Support for depression screening and treatment for children \& adolescents with ADHD. 2009.

14. National Youth Commission in Korea. White paper of Adolescents. 2007.

15. Foreman MD, Wykle M. Nursing standard of practice protocol: sleep disturbance in elderly patients. Geriatr Nurs. 1996;16:238-43.

16. Dewald JF, Meijer AM,Oort FJ, et al. The influence of sleep quality, sleep duration and sleepiness on school performance in children and adolescents: a meta-analytic review. Sleep Med. 2010;14:179-89.

17. Chase JE, Gidal BE. Melatonin: Therapeutic use in sleep disorders. Ann Pharmacother. 1997;31(10):1218-26.

18. Lewy AJ, Sack RL. Exogenous melatonin's phase-shifting effects on the endogenous melatonin profile in sighted humans: a brief review and critique of the literature. J Biol Rhythms. 1997;12(6):588-94.

19. $\mathrm{Xu} \mathrm{Z}, \mathrm{Su} \mathrm{H}, \mathrm{Zou} \mathrm{Y}$, et al. Sleep quality of Chinese adolescents: Distribution and its associated factors. J Paediatr Child Health. 2012;48:138-45.

20. Saxvig IW, Pallesen S, Wilhelmsen LA, et al. Prevalence and correlates of delayed sleep phase in high school students. Sleep Med. 2012;13:193-99.

21. Neder N, Chrousos GP, Kino T. Interactions of the circadian CLOCK system and the HPA axis. Trends Endocrinol Metab. 2010;21(5):277-86.

22. Deuschle M, Schweiger U, Weber B, et al. Diurnal activity and pulsatility of the hypothalamus-pitutary-adrenal system in male depressed patients and healthy controls. J Clin Endocrino Metab. 1997;82:234-238.

23. Yoon HJ, Oh YH, Song JH, et al. Association between the daytime plasma cortisol level and depression component of Hamilton depression rating scale in Korean depression clinic outpatients. J Korean Neuropsychiatr Assoc. 2015;54(1):91-6.

24. Schulz P, Kirschbaum C, Prubne J, et al. Increased free cortisol secretion after awakening in chronically stressed individuals due to work overload. Stress Med. 1998;14:91-7.

25. Pigeon WR, Cerulli C, Richards H, et al. Sleep disturbances and their association with mental health among women exposed to intimate partner violence. J Womens Health (Larchmt). 2011;20:1923-9.

26. Roane BM, Taylor DJ. Adolescent insomnia as a risk factor for early adult depression and substance abuse. Sleep. 2008;31:1351-6.

27. Stores G. Children's sleep disorders: Modern approaches, developmental effects, and children at special risk. Dev Med Child Neurol. 1999;41:568-73.

28. http://www.seoul.co.kr

29. Owens J; Adolescent Sleep Working Group; Committee on Adolescence. Insufficient sleep in adolescents and young adults: An update on causes and consequences. Pediatrics. 2014;134(3):e921-32.

30. Compas BE. Assessment of major and daily stressful events during adolescence: The adolescent perceived events scale. J Consult Clin Psychol. 55(4):534-41.

31. Soo HY. Psychosocial approach to the stress of the youth. Yonsei Social Welfare Review. 1996;3:121-39.

32. http://www.bogun.seoul.kr

33. Seoyoun K, Hyojae K, Min JK, et al. How much does depressive mood affect sleep for high school students? J Sleep Disord Ther. 2016;5:1.

34. Stahl SM. Stahl's psychopharmacology: Neuroscientific basis and practical applications. Cambridge medicine. 4th edition.

35. Pacchierotti C. Melatonin in psychiatric disorders: A review on the melatonin involvement in psychiatry. Front Neuroendocrinol. 2001;22(1):18-32.

36. Arendt J. Importance and relevance of melatonin to human biological rhythms. J Neuroendocrinol. 15(4):427-31.

37. Dijk DJ, Cajochen C. Melatonin and the circardian regulation of sleep initiation, consolidation, structure, and the sleep EEG. J Biol Rhythms. 1997;12(6):627-35.

38. Krauchi K, Wirz JA. Circadian clues to sleep onset mechanisms. Neuropsychopharmacology. 2001;25(5):S92S6.

39. Zhdanova IV, Wurtman RJ, Lynch HJ, et al. Sleepinducing effects of low doses of melatonin ingested in the evening. Clin Pharmacol Ther. 1995;57(5):552-58.

40. March JS, Silva S, Petrycki S, et al. The treatment for adolescents with depression study (TADS): Long-term effectiveness and safety outcomes. Arch Gen Psychiatry. 2008;65(1):101.

41. Baker TL. Introduction to sleep disorder. Med Clin North Am. 1985;1132-52.

42. Fabbian F, Smolensky MH, Tiseo R, et al. Dipper and nondipper blood pressure $24 \mathrm{~h}$ patterns: Circadian rhythmdependent physiologic and pathophysiologic mechanisms. Chronobiol Int. 2013;30:17-30.

43. Lewy A. Melatonin treatment of winter depression: a pilot study. Psychiatry Res. 1998;77(1):57-61.

44. Strickland PL, Deakin JF, Percival C, et al. Bio-scocial origins of depression in the community. Interactions between social adversity, cortisol and serotonin neurotransmission. Br J Psychiatry. 2002;180:168-73. 